\title{
Refractory High Risk Myelodysplastic Syndrome
}

National Cancer Institute

\section{Source}

National Cancer Institute. Refractory High Risk Myelodysplastic Syndrome. NCI

Thesaurus. Code C138964.

High risk myelodysplastic syndrome that does not respond to treatment. 